\title{
Propuesta de inNOVACIÓn PEDAgógica en el Centro Docente San José del MUNICIPIO DE AIPE, HUILA
}

\author{
PROPOSAL FOR PEDAGOGICAL INNOVATION AT THE \\ SAN JOSÉ TEACHING CENTER IN THE MUNICIPALITY \\ OF AIPE, HUILA
}

Ana Elizabeth Lozada Pinto*

Lorena Marcela Castro Aldana**

Tania Lorena Medina Rojas***

\footnotetext{
* Magíster en Educación de la Universidad Surcolombiana. Docente de la Institución Educativa Niño Jesús de Praga de Ibagué. anita.alarcon17@hotmail.es 0000-0002-6119-3112

** Magíster en Educación de la Universidad Surcolombiana. Docente de la Universidad Antonio Nariño. lomaca0809@hotmail.com 0000-0002-0611-0310

*** Magíster en Educación de la Universidad Surcolombiana. Docente de la Institución educativa Chapinero de Neiva. aniat_1990@hotmail.com 0000-0003-2653-6922
}

Cómo citar este artículo: Lozada, P. A. E., Castro, A. L. M. \& Medina, R. T. L. (2021). Propuesta de innovación pedagógica en el Centro Docente San José del municipio de Aipe, Huila. Revista PACA 11, pp. 139-156.
Resumen: El artículo de investigación muestra la experiencia que se originó en la década de los años ochenta en el Centro Docente San José en el municipio de Aipe (Huila), producto de un colectivo de maestros inspirados y motivados por el auge del movimiento pedagógico como respuesta a las reformas educativas del gobierno, que pretendían convertir al maestro en un simple administrador del currículo y reproductor de contenidos. De esta manera se empezó a generar una propuesta alternativa de innovación educativa, inspirada en la pedagogía crítica y la educación popular, abriendo caminos a través de proyectos investigativos, teniendo en cuenta su entorno social y cultural. El objetivo es sistematizar a través de las voces de la comunidad educativa la experiencia. A través de los enfoques cualitativo y crítico-social se busca reconstruir, interpretar y generar nuevas categorías que emergen y que son vitales para potenciar una innovación educativa. Los principales hallazgos de la experiencia superaban la simple reproducción de contenidos que ya vienen definidos y empezaba con el protagonismo de los niños a investigar temas que el contexto demandaba en los entornos de sus barrios, la comunidad, el río, las huertas y situaciones que a cualquier momento podían despertar el interés de los estudiantes con un acompañamiento permanente y reflexivo de los docentes y las familias. 
Palabras clave: propuesta alternativa, sistematización de experiencia, entorno, familias, comunidad.

Abstract: The research article shows the experience that originated in the eighties at the San José Teaching Center in the municipality of Aipe (Huila), the product of a group of teachers inspired and motivated by the rise of the pedagogical movement in response to the government's educational reforms, which sought to turn the teacher into a simple administrator of the curriculum and content player. In this way, an alternative proposal for educational innovation began to be generated, inspired by critical pedagogy and popular education, opening paths through research projects, taking into account its social and cultural environment. The objective is to systematize the experience through the voices of the educational community. Through the qualitative and critical-social approaches, it seeks to reconstruct, interpret and generate new categories that emerge and that are vital to promote educational innovation. The main findings of the experience went beyond the simple reproduction of content that is already defined and began with the protagonism of the children to investigate topics that the context demanded in the environments of their neighborhoods, the community, the river, the orchards and situations that any moment they could awaken the interest of the students with a permanent and reflective accompaniment of the teachers and the families.

Keywords: alternative proposal, systematization of experience, environment, families, community.

\section{Introducción}

En la década del ochenta surge en Colombia el movimiento pedagógico liderado por docentes de la educación básica y superior, como crítica al modelo tecnocrático que pretendía convertir al maestro en un simple administrador del currículo. Fue así como se despertó un interés colectivo de docentes de todas las regiones de Colombia por retomar la pedagogía como escenario para transformar esa antigua escuela bancaria que estaba cayendo en la reproducción de contenidos. Todas estas apuestas y búsquedas del magisterio por transformar las relaciones en el aula estaban inspiradas en la pedagogía crítica y la educación popular, que buscaban definir un proyecto de sociedad más democrático y solidario alrededor de una escuela alternativa.

En ese proceso, un colectivo de cinco maestros constituyó una escuela alternativa en el municipio de Aipe (Huila), proponiendo una 
educación participativa y popular, con la colaboración de la Asociación de Institutores Huilenses (ADIH), sindicato de maestros filial de Fecode, y el acompañamiento pedagógico del Centro de Investigación y Educación Popular (Cinep), que se convirtieron en un soporte para animar este sueño colectivo.

El proyecto, desarrollado, generó un vínculo importante con las familias y algunos sectores de la comunidad tanto urbana como rural, que promovió en los niños la investigación de la realidad y el contexto local para convertirlo en contenidos de aprendizajes. En el Huila, durante los años ochenta fueron muy visibles dos experiencias de innovación educativa que tuvieron resonancia a nivel nacional: de una parte, la experiencia de la Escuela Popular Claretiana, llamada Filo de Hambre, quien tiene cuarenta años de existencia, mantiene su dinámica y trabajo articulado con las familias y la comunidad; de otra parte, la experiencia de la Escuela San José en el municipio de Aipe. Aunque no logró sostenerse en el tiempo, mantiene viva la historia del movimiento pedagógico colombiano, la cual se debe recuperar para analizar su inspiración pedagógica, los aprendizajes y todo su potencial, a fin de ser compartidos en estos tiempos en que se ha venido despojando al maestro de su saber pedagógico, tiempos que algunos teóricos como Luis Bonilla y Marco Raúl Mejía han denominado un apagón pedagógico global, en la que promueven la conciencia de la necesidad de una nueva escuela alejada de la reproducción de contenidos y poco pertinente.

Bajo esta premisa, la investigación se despliega con el interés y la motivación de recuperarla apuesta pedagógica desde las voces de maestros, familia y comunidad, protagonistas del proceso del enfoque innovador, crítico y transformador, con un trabajo que permitió la reconstrucción de sueños y objetivos, así como la producción de conocimientos que aportó al quehacer del maestro, la escuela, la comunidad y el territorio, en relación con la necesidad de cambio en la educación tradicional.

La reconstrucción de esta práctica se enriqueció a través de testimonios, revisión bibliográfica y videos, con el fin de generar información vital para el proceso de recolección y análisis bajo la metodología cualitativa. Entre los resultados obtenidos se encuentran aspectos como el contexto, las influencias y las motivaciones de los protagonistas; los referentes teóricos, pedagógicos y metodológicos que inspiraron la experiencia, y la emergencia de lo comunitario, el territorio y lo pedagógico. 
La Escuela San José tiene todos los méritos en esas innovaciones educativas que promovió el movimiento pedagógico en su tiempo bajo la corriente de una Escuela Nueva. A partir de lo anterior, el presente texto busca sistematizar la experiencia a través de las voces de la comunidad educativa, debido a que las pedagogías que surgen en la educación son difíciles de encontrar y de poner en práctica, sobre todo porque le aportan al cambio. Ello trasciende una serie de corrientes lo cual contribuye a obtener vivencias significativas y a abrir la posibilidad de que los educadores asuman el movimiento de cambio de paradigma de la educación, con el propósito de que los maestros, las familias y la comunidad actúen como verdaderos agentes de renovación educativa.

En ese sentido, se reconoce el trabajo desarrollado en la Escuela San José, mostrando sus aciertos y dificultades, a través de una recontextualización del marco de la educación popular, un modelo de escuela alternativa para esta problemática global desde todas las áreas y niveles educativos. De otra manera, la investigación como estrategia pedagógica es una de las opciones significativas para que el sujeto sea consciente de su realidad social y de un diálogo de saberes que conlleve a superar la fragmentación muy presente en la educación tradicional. En estos tiempos, al revisar el programa Ondas de Colciencias, se encuentra diversidad de estrategias encaminadas a la investigación, que de igual manera proponía la experiencia San José con los niños, acercándose a estos programas institucionales, donde los contextos provocan las preguntas con una participación muy activa del estudiante.

\section{Referentes teóricos}

Teniendo en cuenta el interés por investigar la experiencia de innovación pedagógica realizada en el Centro Docente San José en el municipio de Aipe, durante casi una década de trabajo se pretende analizar diferentes fundamentos teóricos que subyacen en conceptos fundamentales como la recopilación de la experiencia de educación como innovación en la vida.

Seguido a lo anterior, se reconstruye la experiencia, la cual está en la búsqueda de alternativas de escuelas que pretendían convertir al estudiante en un sujeto protagónico de la construcción de los saberes, de estrechar el vínculo con las familias y las comunidades y de construir un currículo que dialogara con el contexto. La experiencia se desarrolló durante 
casi una década en el municipio de Aipe. En relación con lo anterior, según Freire, la educación popular genera un movimiento de ideas, símbolos y representaciones mentales que acompañan y constituyen la protesta, la movilización o la construcción de sentidos alternos a los dominantes. En los movimientos sociales adquieren materialidad los entretejidos que conforman la educación popular en Colombia, los cuales recogen un conjunto de saberes sobre diversos ámbitos o áreas de la educación popular que se han generado y que pasan por lo político, lo pedagógico, las apuestas investigativas, formativas y de sistematización, desde las prácticas educativas y espacios de diferenciación étnica y de género.

Por el contrario, para Célestin Freinet es importante desarrollar una educación auténticamente popular basada en el trabajo. Esta es la actividad central de la que se desprenden todas las adquisiciones del niño. Integrar la escuela a la sociedad y al trabajo le devuelve a la vida y al ambiente. La educación tiene sentido en la colectividad; este es su fin y su medio; por ello, la relación educativa básica no se da entre el maestro y el alumno, sino entre este último y la colectividad (Jiménez, 1985).

De ahí que sea necesario partir del reconocimiento de la importancia, pertinencia y vigencia de la concepción de Freire sobre educación popular como práctica educacional y como una teoría pedagógica para los tiempos presentes, válida en el contexto latinoamericano y extendida incluso al resto del mundo.

Así mismo, este autor proporciona conocimientos que permiten pasar de una agrupación a un trabajo en equipo (cooperativo). Es por ello que los alumnos, educadores y niños producen trabajo significativo y cooperativo. El punto de partida es la realidad global y totalizante; de ahí esta pedagogía conlleva el análisis crítico de la realidad, la búsqueda de las múltiples relaciones e implicaciones, la exigencia de la investigación y el respeto al derecho que tiene toda persona a experimentar, a emitir hipótesis, a cometer errores, a cuestionar el pasado, a interrogar sobre su ambiente y sobre los mecanismos socioeconómicos. En otras palabras, el derecho al tanteo experimental es un proceso integrado a la formación de la personalidad del niño.

En la pedagogía de Célestin Freinet, el ser humano nace ya con capacidades específicas desarrolladas para desenvolverse en un medio. 
Se ha deducido que la diferencia entre un niño y un adulto son los grados de aprendizaje, errores y experiencias obtenidos a lo largo de su vida, con lo cual se ha dejado de lado la idea de que el niño es un ser imperfecto, indefenso, que no es capaz de aprender por sí solo y debe cambiar según el pensamiento de la sociedad. Esto demuestra que el ser humano en general, sin importar su edad, comete errores y ello no significa que sea ignorante, sino que aprenderá de aquellos. Trayendo a colación a Jiménez (1985), "el niño es como un árbol que no ha terminado todavía su crecimiento, pero se nutre, crece y se defiende exactamente como el árbol adulto" (p. 44).

De acuerdo con Freinet, la experiencia de Aipe se articula en "tanteo experimental", considerando que los aprendizajes se efectúan a partir de las propias experiencias, de la manipulación de la realidad, de la expresión de sus vivencias, de la organización de un contexto próximo, de un ambiente en el que los alumnos puedan formular y expresar sus experiencias (Infante, 2014, p. 6).

Además de todo lo anterior, la pedagogía de Freinet busca fomentar en el niño la autoevaluación, brindándole la oportunidad de aprender de sus errores y encontrarles solución. Para ello se usan ficheros autocorrectores, una especie de plantillas que indica las tareas por realizar, donde los alumnos anotan los niveles alcanzados y las dificultades.

De esta manera, la pedagogía del académico brasileño Freire se convierte en una pedagogía crítica, liberadora y problematizadora del ámbito educativo y social, y ofrece una propuesta revolucionaria, en tanto el sujeto educando, mediante su participación, se convierte en actor indispensable al redimensionar la realidad social que le es propia (Brito, 2014).

Las dimensiones de la propuesta participativa en el proceso educativo educador/educando constituyen un espacio común en el proceso de aprendizaje social: la responsabilidad, la percepción, la comprensión y el conocimiento de la realidad se convierten en un hecho compartido. No obstante, el hecho de ser un proceso gradual de aprendizaje mutuo demanda necesariamente la transformación y el cambio, donde los educandos constituyen actores esenciales (Brito, 2014). 
"Esta enseñanza y este aprendizaje tienen que partir, sin embargo, de los 'condenados de la Tierra', de los oprimidos, de los desarrapados del mundo y de que ellos realmente se solidaricen" (Freire, 1994, p. 2).

Por lo tanto, la teoría de Paulo Freire inspiró a añadir insumos de Célestin Freinet, que aportaron con las técnicas para edificar la propuesta y llevar a cabo los objetivos de la educación popular en el Centro Docente San José. Aún no se contaba con la infraestructura adecuada, apenas en construcción; había sido abandonada por el gobierno nacional. Sin embargo, esta necesidad fue el motor que permitió ver el horizonte de una escuela inclusiva, donde el espacio no fuera obstáculo sino que, por el contrario, se convirtió en la oportunidad de muchos aprendizajes.

La teoría de Paulo Freire se basa en el diálogo propuesto, no se limita ni se reduce a un espacio de cuatro paredes, abarca el conjunto de la comunidad de aprendizaje, incluyendo padres, madres y otros agentes, quienes inciden significativamente $y$, por ende, se debe planear en conjunto (García \& Mallart, 1998). El autor hace énfasis en la democracia como un proceso de participación en el que todos los agentes se consideran iguales; pero, en la práctica la educación anula este desarrollo creando niños, niñas y jóvenes débiles. En ese sentido, la apuesta de Aipe permitía generar procesos de autonomía, interés y participación activa, formando seres conscientes de la realidad, creativos, reflexivos e investigadores en su contexto.

Desde la propuesta pedagógica freireina, los nuevos procesos formativos deben generar un "hombre nuevo", consciente de su realidad y comprometido con su transformación, resaltando la construcción dialógica, el humanismo crítico, las prácticas emancipadoras y la reinvención como aspectos fundamentales para la constitución de vínculos sociales solidarios (Ortega, 2014).

Según Jean Piaget, el desarrollo intelectual es un proceso de reestructuración del conocimiento; inicia con un cambio externo, creando un conflicto o desequilibrio en la persona, el cual modifica la estructura existente, elaborando nuevas ideas o esquemas, a medida que el humano progresa. 
Cada estadio, según la teoría de Piaget, sufre límites de edad en el que pueden variar en los distintos grupos poblacionales, de acuerdo con el contexto en el cual se desarrolle su formación, la cultura, etc. Las adquisiciones cognitivas en cada estadio no son productos intelectuales aislados, sino que guardan una estrecha relación (Saldarriaga, Bravo \& Loor-Rivadeneira, 2016).

Se realizó un acercamiento al pensamiento de Jean Piaget, el cual contribuyó a evidenciar el desarrollo cognitivo de los niños y niñas; a su vez buscó fomentar el máximo de sus posibilidades de pensamiento. Para ello se hizo indispensable contar con la manera concreta como el niño desarrollaba sus tareas, para determinar en qué estadio se encontraba, a partir de sus condiciones concretas y no de las planteada en el currículum o el grado escolar en el que se encontraba (Mejía, 1987, p. 40). Para el caso de la experiencia en Aipe, se inició con un diagnóstico para reconocer las habilidades y capacidades de los estudiantes, con el propósito de asignar tareas que demostraran el aprendizaje y la interiorización como un hecho concreto.

Para este autor, el pensamiento no es solo la actividad lingüística para la adquisición de saberes; también resalta el desarrollo de imágenes, acciones, juegos, dibujos y todo lo concierne a una comprensión real; por ende, en esta experiencia se identificaron seis elementos esenciales para el aprendizaje en relación con lo que se quiso reconocer en el niño.

Por estas razones, la experiencia del Centro Docente San José de Aipe tomó elementos de la teoría piagetiana, en la búsqueda de una educación que desarrollara en los niños y las niñas el máximo de sus posibilidades de pensamiento, teniendo en cuenta los estadios del desarrollo generados en la construcción psicológica del aprendizaje del infante.

Por otra parte, encontramos la psicología de Vygotsky, quien pondera y reconoce la actividad del sujeto, quien no se limita a responder a los estímulos, sino que usa su actividad para transformarlos. Para llegar a la modificación de los estímulos, el sujeto usa instrumentos mediadores. La cultura proporciona las herramientas necesarias para poder modificar el entorno; por ende, para Vygotsky el contexto social influye en el aprendizaje, las actitudes y las creencias; tiene una profunda influencia en cómo se piensa. El contexto forma parte del proceso de desarrollo y, en tanto tal, moldea los procesos cognitivos. 
Para Vygotsky, el pensamiento del niño se va estructurando de forma gradual, y la maduración influye en que el niño pueda hacer ciertas cosas o no, por lo que considera que hay requisitos de maduración para determinar ciertos logros cognitivos, pero esta maduración no necesariamente determina el desarrollo. No solo el desarrollo puede afectar el aprendizaje, sino que el aprendizaje también puede afectar el desarrollo; todo depende de las relaciones entre el niño y su entorno. Por ello, debe considerarse el nivel de avance del niño, pero también presentarle información que siga propiciándole el avance en su desarrollo (Brodova \& Lions, como se citó en Martínez, 2008).

Vygotsky y los aportes socioculturales alimentaron las construcciones de esta experiencia sobre las estructuras mentales en cuanto a las mediaciones simbólicas, las cuales "se desarrollan con la interacción del sujeto tiene con su entorno inmediato. Toda función va a aparecer en dos planos: en lo social (interpsíquico) y en lo psicológico (intrapsíquico)" (Vygotsky, 1978). De este autor se toman los conceptos de contexto y cultura, para generar propuestas de transformación de los entornos, los sujetos y las prácticas cotidianas, a partir de metodologías participativas y del análisis de la sociedad.

Vygotsky considera cinco conceptos fundamentales: las funciones mentales, las habilidades psicológicas, la zona de desarrollo próximo, las herramientas del pensamiento y la mediación. Las influencias en las relaciones sociales y las actividades orientadas al aprendizaje de los niños incorporan a su pensamiento herramientas culturales como el lenguaje, los sistemas de conteo, la escritura, el arte y otras invenciones sociales, como se evidencia en la metodología aplicada en la experiencia.

Con esta revisión se concluye una contextualización mediante la pedagogía de diferentes teóricos como Célestin Freinet, Jean Piaget, Paulo Freire, Vygotsky, nutriéndola teóricamente con los marcos de la educación popular, la pedagogía crítica, el desarrollo intelectual, el tanteo experimental, el trabajo cooperativo, el proceso formativo, el diálogo propuesto, el desarrollo cognitivo de los niños y niñas, los aportes socioculturales (contexto), etc.; cuyo mayor exponente es Paulo Freire. El trabajo se adaptó a través de las estrategias e instrumentos, teniendo en cuenta los recursos que les brindaba su medio en ese momento.

En este proyecto se evidenció que, gracias al trabajo de estos docentes y diferentes teóricos ya nombrados, se observó un cambio visible en el 
distintivo de los niños (más creativos, críticos, seguros, responsables, analíticos, espontáneos, con capacidad para relacionarse y organizarse grupalmente, innovadores, investigadores, etc.).

\section{Metodología}

Este proyecto de investigación pretende sistematizar a través de las voces de la comunidad educativa la experiencia inspirada en los principios de la pedagogía crítica y la educación popular, la cual tuvo un cierto reconocimiento a nivel regional y nacional; por tal razón, consintió el registro de las narraciones mediante un proceso cualitativo en que permitió entender sentimientos, emociones y evocación de recuerdos significativos del protagonismo que se dio en ese contexto.

La investigación se realizó de manera transversal, retrospectiva y de sistematización, la cual ofreció resultados descriptivos de un momento determinado. Además, la sistematización permitió rescatar elementos importantes dentro del proceso, comprender la dinámica y la función en las que se desarrolló en el contexto.

Por lo anterior fue clave la recolección y revisión de varios insumos como la revista Educación y Cultura, número 10, de Fecode, el artículo Aipe: Buscando caminos alternativos en educación (Perdomo, Morales, Celis, Gómez \& Guerrero, 1986); el libro Los maestros construimos futuro: experiencias pedagógicas en Educación Formal (Cinep, Cepecs \& IPC, 1990); el análisis y contextualización del video Con la raaa... de árbol (Bermúdez, 1988), entre otros videos relacionados con la experiencia de Aipe; y las Cartas Pedagógicas (Mejía, 1987), los cuales sirvieron para plasmar y analizar la información encontrada de manera ordenada y sin dejar por fuera elementos importantes.

Además, se realizaron entrevistas, las cuales fueron grabadas y sistematizadas para lograr un panorama general de percepciones sobre la experiencia del Centro Docente San José. Estos aportes contribuyeron a la memoria de la experiencia y a la reflexión de la práctica. Los datos recogidos fueron muy relevantes para la investigación, pues gracias a ellos se logró reconstruir una vasta información de la experiencia, la cual no había sido documentada; por ello, se contó con la participación de algunos profesores y con un grupo de exalumnos del Centro Docente San José; también se tuvieron en cuenta varias personas de la comunidad educativa. 
Dicho proceso se desarrolló en tres fases, las cuales facilitaron su sistematización y documentación:

Primera fase: Reconstrucción de la experiencia. El objetivo primordial fue ordenar las reflexiones y sentidos de los actores a la experiencia, con el fin de obtener los momentos más significativos de la práctica, determinando el contexto, la caracterización y el sentido que encuentran los actores, para comprender e interpretar el proceso de iniciación de la experiencia, a través de los artículos escritos por los pioneros de la experiencia, y así mismo conseguir un acercamiento con los participantes en este proyecto.

Segunda fase: Reflexión de la experiencia. En esta fase se interpreta la comprensión de la experiencia, los aprendizajes y las emergencias de nuevos referentes, que pueden ser muy potentes para pensar en propuestas de escuelas alternativas.

Tercera fase: Resignificación y potenciación de la experiencia. En esta fase se determinan las fortalezas y los obstáculos del desarrollo de la experiencia, así como las nuevas categorías que emergen de la experiencia y contribuyen a su resignificación y potenciación como referente para estos nuevos tiempos de recuperación de los saberes pedagógicos de los maestros.

\section{Resultados}

A inicios de los años ochenta, con la inspiración que provocaba el movimiento pedagógico que empezaba a tomar más forma y con el propósito de articular una escuela a los procesos organizativos y a los movimientos sociales, un colectivo de docentes se traslada al municipio de Aipe para hacer posible el sueño de una escuela nueva y alternativa, un proyecto desafiante el cual implicaba pensar no en la continuación de reproducción de contenidos, sino en construirlos junto con los estudiantes de acuerdo con las condiciones del contexto y las características del medio social.

Una iniciativa atrevida que proponía distanciarse de la práctica instrumental de transmitir información. Este proceso, liderado por el docente Luis Felipe Celis Tovar, la profesora Gala Georgina Guerrero 
Valencia y Marco Raúl Mejía Jiménez, del Cinep, fue una experiencia que duró aproximadamente diez años hasta que gradualmente se fue disolviendo por el fallecimiento, traslado y jubilación de los profesores. Sin embargo, algunos docentes mantuvieron su práctica de manera individual.

Actualmente, los docentes Luis Felipe Celis Tovar, la profesora Gala Georgina Guerrero Valencia y el Asesor de Cinep de esa época, Marco Raúl Mejía Jiménez, participantes en esta propuesta alternativa, ven con satisfacción cómo la experiencia ha cruzado fronteras y ha tenido un reconocimiento importante en el sector educativo, gracias a su manera de trabajar la indagación con los niños y las niñas y a las iniciativas de investigación. Por ejemplo, el video Con la raaa... de árbol se presentó en varios encuentros académicos nacionales e internacionales de Latinoamérica como simposios, conferencias, diálogos, tertulias, cafés pedagógicos, debates, entre otros, a fin de compartir los saberes adquiridos.

También, cuando se revisa el proceso metodológico del programa Ondas, son muchas las similitudes que se encuentran con la experiencia de San José en todo el proceso de investigación promovido con niños y jóvenes. La necesidad de la pregunta, la formulación del problema, la planeación para buscar la información, la realización de análisis y la socialización de los resultados con las familias apoyándose en el arte se evidencian, por ejemplo, en el video Con la raaa...de árbol, que proporcionó la ruta de investigación como estrategia pedagógica.

Por consiguiente, estos profesores mencionan que la experiencia fue un ejercicio de trabajo maravilloso e inolvidable en sus vidas; cada uno descubrió habilidades y conceptos de formación pedagógica que desconocían debido al sistema tradicionalista. Estos docentes están seguros de haber dejado una huella significativa en sus exalumnos, familias y comunidad en general

"Esto es lo bello que queda, el vínculo y el cariño que le brindan a uno los que pasaron por este proceso y guardan recuerdos de las salidas, de las visitas. Muchos de ellos son profesionales, emprendedores, con muchas capacidades y que siempre guardan afecto donde uno se los encuentre" (Luis Felipe Celis Tovar, Docente). 
"Esto fue un ejercicio muy bonito y Gala fue una mujer maravillosa, los que vivieron la experiencia con ella, su paciencia, su aprendizaje, las enojadas; pero todo tratando de construir el proyecto. Además, el equipo era un grupo maravillosísimo, amoroso, fue un tiempo maravilloso con todos" (Marco Raúl Mejía, Asesor).

"Siempre mantuve el entusiasmo hasta el día que decidí retirarme, luego de haber adquirido la pensión. Lo triste es cuando queda uno como solo. La fuerza de estas experiencias es cuando se trabaja en colectivo, con compañeros y compañeras que son comprometidos. $Y$ eso es lo que debe pasar si queremos cambiar la educación, que se comparta en el grupo, se reflexione, se miren los aprendizajes, las dificultades, ese diálogo es fundamental. Pero si cada maestro está por su lado haciendo cosas sin la reflexión colectiva no vamos a avanzar. Y eso es lo que me preocupa" (Gala Guerrero, Docente).

Durante el proceso de análisis e interpretación de la información obtenida, mediante la aplicación de técnicas e instrumentos para la recolección de la información; entrevistas a profundidad y revisión de la literatura pertinente (videos, documentos), en un sentido más amplio, se pretende mostrar los hallazgos de esta investigación que, en su primer momento, demostró cómo el contexto, las influencias y las motivaciones de los protagonistas fueron vitales para el surgimiento de esta experiencia pedagógica, puesto que la crisis una vez más reflejó las fracturas de los ámbitos políticos, sociales, económicos y educativos y, más aún, en este último, el Estado busca homogenizar los aprendizajes, desconociendo la historia, los lugares e intereses de los individuos.

La influencia del contexto a nivel nacional y su crisis social, generó el congreso de Fecode, el cual se realizó a finales de los años setenta en Neiva, donde se hizo el lanzamiento del proyecto Nueva Escuela, como propuesta que buscaba transformar la democracia desde la escuela, para contribuir a las transformaciones que necesitaba Colombia en todos los órdenes sociales y económicos, y de esa manera contrarrestar la otra consigna que sectores radicales de Fecode proponían: que solo si cambiaba el sistema, cambiaría la educación; que solo sobre las ruinas del Estado burgués nacería el Estado proletario y, por lo tanto, el sindicato y la escuela estarían al servicio de esta causa; tiempo después, en el municipio de Rivera, en una finca llamada La Hondita, se puede decir que nace el 
movimiento pedagógico previo al congreso de Bucaramanga en el 82, que es donde definitivamente se hace el lanzamiento. Personajes como Abel Rodríguez, Felipe Rojas, Martha Cárdenas, William Sánchez y Marco Raúl Mejía, quienes venía del Cinep, organizan las bases del movimiento. Así, se lanza la consigna "Educar y luchar por la liberación nacional", un movimiento que recupera la condición intelectual del maestro. Se funda la revista Educación y Cultura y los CEID (Centro de Estudios e Investigaciones Docentes) como centros dinamizadores del pensamiento pedagógico. Como impulsábamos la propuesta de Escuela Nueva, ciertos sectores radicales de Fecode nos decían "los pedagogicistas", "los de las rondas infantiles", "rin rin renacuajo", "los reformistas de la educación", que éramos enemigos de la revolución porque estábamos desviando la atención de lo que debería ser la lucha sindical, que era cambiar el sistema para cambiar la escuela.

En este orden de ideas, el movimiento pedagógico en su primer número de la revista Educación y Cultura (1984) constituyó la fuente de inspiración para colectivos de maestros por innovar y pensar una escuela alternativa. Eso fue precisamente lo que sucedió en Aipe con el Centro Docente San José, en tanto el magisterio no se limitó a mantener una posición contestataria en rechazo a la política educativa del ministerio, sino que se formularon propuestas sobre nuevas formas de hacer escuelas y de ser maestros.

Además, la presencia de las corrientes religiosas en la vida de los protagonistas fue vital para asumir retos como estos; las interacciones alimentaron el don del servicio a la comunidad, la colaboración, el respeto y la asertividad en la resolución de las problemáticas sociales, premisas que fueron un buen augurio para consolidar las bases sobre educación popular y los objetivos de este tipo de sistema; por tal razón, este colectivo de docentes tuvo plena disposición a promover cambios en la escuela y, con ello, un cambio en la sociedad para la transformación de las relaciones de desigualdad y de injustica, ligadas a sus experiencias vitales con sus familias de origen católico y con unas prácticas de generosidad y altruismo. Igualmente, sus acercamientos a movimientos políticos de izquierda y a la teología de la liberación.

Por otro lado, la metodología impartida en esta experiencia se basó en las Cartas Pedagógicas, las cuales se originaron por la necesidad de 
mantener una comunicación entre los docentes y el asesor, en este caso Marco Raúl Mejía. Desde el Cinep acompañaban este propósito de dar pautas a las actividades, las cuales fueron conducidas por referentes de la Escuela Nueva, en los se tenían en cuenta los intereses de los estudiantes y las dificultades que se presentaban en aprendizajes asociados a lectoescritura, matemáticas, etc. Simultáneamente, las inquietudes de los docentes también fueron indispensables para dar inicio a un trabajo colectivo que conllevaría a nutrir saberes que permitieran hacer cohesión entre teoría y práctica.

La creación de las Cartas Pedagógicas compartía las reflexiones de las jornadas escolares y se retroalimentaban a partir de la discusión que se tenía con los profesores, basados en materiales de Freinet, Freire, Piaget y Vygotsky. Después, las guías fueron totalmente propias. Con estas cartas se abrió paso a una nueva perspectiva de orientación al maestro, pues con ellas se asesoró el trabajo del proyecto de educación popular en el Centro Docente San José, dando explicación a metodologías, teorías y recursos que permitieran al docente dar una posible solución a los problemas sociales, ambientales, espirituales, formativos, económicos y políticos que se estaban viviendo en el municipio. Por ende, fue un instrumento pedagógico que permitió superar dichas deficiencias y que, a su vez, potencializó y desarrolló capacidades en la práctica pedagógica.

Además, este instrumento fue evidencia de las luchas contra los excesivos conocimientos que se dieron en ese tiempo, donde las capacitaciones no tenían ninguna vinculación con la práctica de ser maestro. Por eso, esta asesoría pretendía ayudar a solucionar problemas específicos de la actividad. De esta manera se eliminaron los costos que representaba el desplazamiento del asesor, los materiales y tiempos de trabajo, bajo un sistema de correspondencia que les permitía a los docentes expresar sus inquietudes y el asesor respondía dando algunas pautas y orientaciones. Desde luego, el asesor también hacía presencia en campo en determinados momentos, para retroalimentar la experiencia.

Dentro del proceso pedagógico en el aula de clase, el trabajo escolar en el Centro Docente San José se evidenció de la siguiente manera:

- El niño reconoce su medio.

- Los niños discuten su medio y se interesan, proponiendo y fijándose temas para investigar. 
- El niño investiga (primer paso de interiorización) su medio.

- El niño confronta su percepción del medio (puesta en común), mediante el diálogo directo y representando.

- El niño critica las otras percepciones y la suya propia.

- El niño relaciona con otros medios (conocimientos generales, ciencia, integración de áreas, participación del maestro, etc.).

- El niño colectiviza conocimiento al compartir sus relaciones generales aprendidas a través de manualidades, representaciones, juegos.

- El niño deriva acciones hacia el contexto social, a partir de elementos ganados en el proceso de construcción de conocimientos.

- Los niños discuten nuevos temas de investigación, los organizan colectivamente e inician nuevamente la investigación.

- Se van generando procesos acabados con productos terminados como cartillas y representaciones las cuales se llevan a la comunidad y a la familia (Perdomo, Morales, Celis, Gómez \& Guerrero, 1986).

En lo anterior, este paso a paso permitió la apertura a los centros de interés y unidades temáticas, que materializaban, exploraban y transversalizaban todos los ámbitos del ser humano.

Por último, es de resaltar el esfuerzo del Centro Docente San José por contribuir a construir un sentimiento de comunidad, fortaleciendo los vínculos y los lazos sociales frente al embate del individualismo, el desarraigo y la indiferencia con el destino del otro, característicos de estos tiempos. Por eso, valoramos el sentido de lo comunitario que, según Zibechi (2019, p. 61) "se construye a través del hacer colectivo de varones y mujeres, niñas, niños y ancianos, quienes al trabajar reunidos hacen comunidad, hacen lo común. Reducir la comunidad a institución, hecha e instituida de una vez y para siempre, oculta los trabajos colectivos que son los que generan vida, sentido, forma y fondo al hecho comunal".

\section{Conclusiones}

En relación con el objetivo general, al sistematizar la experiencia del proyecto Educativo Alternativo del Centro Docente San José, se pudo evidenciar a través de las voces de sus protagonistas. Se trató de una propuesta de innovación pedagógica, se descubrieron a lo largo de esta investigación logros y algunas limitaciones. 
Igualmente, queremos destacar la importancia que constituyó este aporte desde la educación popular, implementado en una escuela formal, considerando y enfatizando que esta experiencia abordó la búsqueda de una nueva educación. Se desarrolló mediante la pedagogía de Freinet, nutriéndola teóricamente con los marcos de la educación popular y de la pedagogía crítica, cuyo mayor exponente es Paulo Freire. Esto quiere decir que el trabajo se adaptó a las estrategias e instrumentos, teniendo en cuenta los recursos que le brindaba el medio en ese momento.

Durante la interacción con los entrevistados se evidenciaron ciertas acciones de integración con diferentes grupos sociales, como el eje orientador de las iniciativas de la escuela u organizaciones de la comunidad, como se logró en el sector social y económico, acciones que tenían como función convocar, apoyar, informar y motivar para cumplir dicho trabajo.

Se demostró una buena participación de las familias, se promovió un mejor bienestar $y$ se fortalecieron capacidades $y$ habilidades comunicativas, interpretativas, argumentativas y compresivas, tanto en los estudiantes como en los padres y madres de familia y docentes.

De igual forma se avanzó en una mayor integración curricular, vinculando la vida real de los niños, actualizando el campo teórico, metodológico y didáctico con los objetivos de la educación popular, y convirtiendo los problemas de la comunidad en temas o unidades temáticas en diferentes áreas, a fin de ser transformadas y estructuradas integralmente, lo que impulsó la reflexión permanente sobre la práctica, haciendo énfasis en los procesos de aprendizaje de los estudiantes y docentes, y la elaboraron de técnicas e instrumentos propios de trabajo (guías, fichas, mapas, carteleras, cartillas, etc.), integrándolos en las diferentes áreas de estudio.

Este proyecto además recolectó el saber, el aprender y el conocer, con la adquisición de valores humanos, siendo favorable para la vida de estos exalumnos, pues hoy son personas que se caracterizan por ser emprendedores, sociables, con sentido artístico e investigativo, con intereses culturales y descubrieron habilidades y gustos para su formación personal y profesional.

En pocas palabras, este proyecto de innovación se fortaleció en manos de estos docentes que amaban su vocación y buscaban cambios para 
mejorar la calidad educativa; docentes que se le medían a todo, con tal de buscar nuevas estrategias, nuevas enseñanzas, nuevas actividades y nuevas sensibilidades.

Por lo anterior, se considera que esta experiencia sigue viva, activa y permanente, pues coincide con la mayor parte de los lineamientos del programa Ondas, relacionados con los pasos metodológicos con los cuales se construyó el proyecto del Centro Docente San José en los años ochenta del siglo pasado.

En definitiva, es muy importante reconocer que esta experiencia abre una oportunidad para pensar y trabajar por una nueva manera de hacer escuela y de ser docente, ahora que se está reactivando el movimiento pedagógico con una propuesta de transformación social. En la pedagogía está en juego un nuevo proyecto de humanidad, que requiere con urgencia una transición ante esta profunda crisis civilizatoria.

\section{Referencias Bibliográficas}

Bermúdez, B. (1988). Con la raaa... de árbol [video]. YouTube. https://www.youtube.com/watch?v=fXfZE2ZB2gM\&t=28s\&ab_ channel=beatrizbermudez

Cinep, Cepecs \& IPC. (1990). Los maestros construimos futuro: experiencias pedagógicas en Educación Formal. Cinep; Cepecs; IPC.

Colectivo Escuela Popular Claretiana. (1987). Filodehambre, una experiencia popular de innovación educativa. Presencia.

Mejía, M. R. (1987). Cartas Pedagógicas. Cinep.

Infante, E. V. (2014). Las teorías de Freinet en la escuela (Tesis doctoral, Universidad Camilo José Cela).

Ortega Valencia, P. (2014). Pedagogía crítica en Colombia: Un estudio en la escuela de sectores populares. Sophia, 10(2), 50-63.

Perdomo, F., Morales, M., Celis, L., Gómez, D. \& Guerrero, G. (1986). Aipe, buscando caminos alternativos en educación. Educación y Cultura, 10, 23-26. $\mathrm{http}: / / \mathrm{www}$. fecoderevistaeducacionycultura.com/images/revistas_1-100/ Educacion\%20y\%20Cultura\%2010.pdf

Saldarriaga-Zambrano, P. J., Bravo-Cedeño, G. D. R. \& Loor-Rivadeneira, M. R. (2016). La teoría constructivista de Jean Piaget y su significación para la pedagogía contemporánea. Dominio de las Ciencias, 2 (3 Especial), 127137.

Zibechi, R. (2019). Los trabajos colectivos como bienes comunes material/ simbólicos. El Apantle/Revista de Estudios Comunitarios, (1). 